\title{
Cortex proliferation
}

\section{Simple phenotype, complex regulatory mechanisms}

\author{
Hongchang Cui and Philip N. Benfey* \\ Biology Department; and IGSP Center for Systems Biology; Duke University; Durham, NC USA
}

Abbreviations: SCR, scarecrow; SHR, short-root; GA, gibberellic acid; SPY, spindly; SLY, sleepy; SNE, sneezy; Pac, paclobutrazol; TSA, trichostatin A; LHP1, like heterochromatin protein 1

Key words: phytohormone signaling, GA, SCR, SPY, SLY, SNE, LHP1, epigenetic, cortex proliferation, arabidopsis

In plants, the cortex is a relatively undifferentiated cell type. Proliferation of cortex tissues initially appeared to be a simple process of repetitive cell division. However, our recent studies showed that in the Arabidopsis root cortex proliferation involves complex regulatory mechanisms. First, it requires the combined activity of the transcriptional regulators SHORT-ROOT (SHR) and SCARECROW (SCR), but SCR also plays a role in restricting the number of cell divisions. The two opposing activities appear to be mediated by different domains of SCR through physical interaction with distinct partners, and whether SCR behaves as an activator or repressor depends on the relative level of the two protein complexes. We confirmed previous findings that GA plays a major role in cortex proliferation, but also found distinct roles for GA signaling components in this process. We showed that $\mathrm{ABA}$ and ethylene also play a role in cortex proliferation, but in an unexpected manner. Finally, we identified an epigenetic component of the regulation, and our data suggested that this is likely the common basis on which various pathways converge. There is evidence that similar mechanisms to those found in Arabidopsis are employed in other plant species.

\section{Introduction}

Regulated cell division is critical for normal growth and development of multi-cellular organisms, as it affects organ size and shape. In plants, the cortex is a relatively undifferentiated cell type and is part of what is called the ground tissue. The number of cortex cell layers varies among species and developmental stages,

*Correspondence to: Philip N. Benfey; Biology Department and IGSP Center for Systems Biology; Duke University; Box 90338; Durham, NC 27708 USA; Tel. 919.660.7338; Fax: 919.613.8177; Email: philip.benfey@duke.edu

Submitted: 04/14/09; Accepted: 04/15/09

Previously published online as a Plant Signaling \& Behavior E-publication: http://www.landesbioscience.com/journals/psb/article/8731

Addendum to: Cui H, Benfey PN. Interplay between SCARECROW, GA and LIKE HETEROCHROMATIN PROTEIN 1 in ground tissue patterning in the Arabidopsis root. Plant J 2009; In press; PMID: 19228333; DOI: 10.1111/i.1365313X.2009.03839.x. but remains almost constant for a particular species at a specific developmental stage. This suggests the existence of a mechanism that strictly regulates the number of cell divisions during plant morphogenesis. Although seemingly a simple process, our recent studies on the Arabidopsis root $^{1}$ showed that cortex proliferation involves complex regulatory mechanisms, ranging from plant hormone signaling to epigenetic regulation.

\section{SCR has Dual Activity in Cortex Proliferation}

In the Arabidopsis root, the cortex and endodermis are derived from the cortex/endodermis initial cell (CEI), through a longitudinal asymmetric cell division. ${ }^{2}$ Initially there is only one cortex cell layer, but later (about 2 weeks after germination) the endodermis undergoes another round of asymmetric cell division, giving rise to a second layer of cortex, termed middle cortex (MC). ${ }^{3}$ Two transcriptional regulators, SHORT-ROOT (SHR) and SCARECROW (SCR), are essential for the longitudinal asymmetric cell divisions. ${ }^{4,5}$ SCR also has a role in suppressing the second division. ${ }^{3}$ Our data suggested that SCR executes these two opposing functions by interacting with different partners through different domains - it activates cell division by interacting with SHR through its central domain, but represses subsequent cell divisions by forming a complex with the transcriptional repressor LIKE HETEROCHROMATIN PROTEIN 1 (LHP1) ${ }^{6,7}$ through its $\mathrm{N}$-terminus, which has a strong nuclear localization signal.

Conceivably, whether SCR behaves as an activator or repressor could be determined by the ratio between the concentrations of the SCR/LHP1 and SHR/SCR complexes. Since SCR transcription is under the control of a feedback loop that depends on the SHR/ SCR complex and is reset in every nascent CEI, ${ }^{8}$ the ratio would not be a constant in all endodermal cells; rather, it is predicted to form a gradient along the root longitudinal axis-lowest at the root tip when the feedback loop is initiated, and higher in more differentiated cells with the buildup of SCR protein, as depicted in Figure 1A. As a consequence, initially SCR would behave as an activator, but above a certain threshold it would turn into a repressor. The expression pattern of $M G P$, a common target of SCR and LHP1, is consistent with this model (Fig. 1B). 


\section{GA has a Major Role in Cortex Proliferation}

An important role for the phytohormone Gibberellin (GA) in cortex proliferation was identified in a previous study, which showed that exogenously applied GA delays MC formation, whereas Paclobutrazol (Pac), a GA biosynthesis inhibitor, enhances the precocious MC phenotype. ${ }^{3}$ They also showed that the GA-deficient mutant, gal-3, has an earlier onset of MC formation. Extending these findings, we found that other GA signaling mutants such as gid 1 and $\mathrm{rga} \Delta 17$ also have the premature MC phenotype. Moreover, GA appears to have a dominant role over SCR and LHP1, as exogenous GA suppresses the MC phenotype in both the $s c r$ and $\operatorname{lhp} 1$ mutants.

\section{Distinct Roles of GA Signaling Components in Cortex Proliferation}

Although GA generally suppresses cortex cell division, our studies also revealed distinct roles for GA signaling components in cortex proliferation. For example, the sleepy $(s l y)$, and the sneezy (sne) mutants, in which DELLA proteins that block GA signaling cannot be degraded, ${ }^{9}$ did not produce MC precociously. Since $S L Y$ and $S N E$ are the closest homologs in the Arabidopis genome, the lack of a MC phenotype could be due to gene redundancy. To investigate this possibility, we generated sly1-10 sne-1 double mutant. However, we detected no root radial pattern defects in the mutant (data now shown), suggesting that they might not be involved in cortex proliferation. Intriguingly, we found a severe MC phenotype in the spindly (spy) mutant. This is unexpected, as the spy mutant has enhanced GA signaling. ${ }^{10}$ The MC phenotype in the spy mutant was not rescued by GA treatment, suggesting that the MC phenotype in the spy mutant is likely due to a defect in GA signaling.

\section{Interplay between Different Phytohormones in Cortex Proliferation}

Since ABA generally acts as an antagonist of GA signaling in plant growth and development, we next determined its role in cortex proliferation. Surprisingly, we found that MC formation is not enhanced, but rather delayed, by ABA. Recently we found that the eto1 mutant, which manifests enhanced ethylene signaling, ${ }^{11}$ also has a premature MC phenotype (Fig. 2). This result suggests that ethylene signaling may play a role in cortex proliferation.

\section{Epigenetic Regulation of Cortex Proliferation}

Another key component of the mechanism regulating cortex proliferation uncovered in our studies is epigenetic regulation. This is obviously the case for the SCR/LHP1 pathway, but is also likely the molecular basis for SPY in cortex cell division. In animals, the SPY homolog was found in a repressor complex that contains histone deacetylases (HDAC) ${ }^{12}$ raising the possibility that SPY may also modulate the activity of HDAC in plants. Supporting this hypothesis, we observed premature MC formation in wild-type Arabidopsis roots treated with Trichostatin A (TSA),
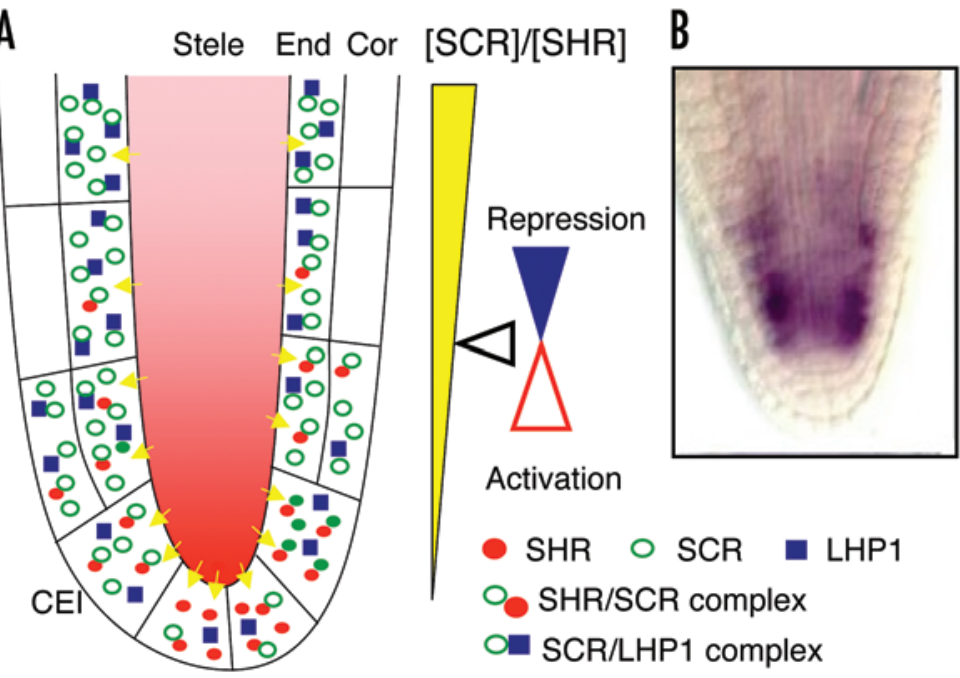

Activation

SHR O SCR

LHP1

OSHR/SCR complex

On SCR/LHP1 complex

Figure 1. SCR activates first, but represses subsequent, asymmetric cell divisions in (A) Schematic depicting the spatial distribution of SHR/SCR SCR/LHP1 complexes along the longitudinal axis of the Arabidopsis root. The between SCR/SHR is low in CEl, favoring formation of the SHR/SCR activar complex; the ratio would increase rapidly due to SHR/SCR dependent positive feedback regulation of SCR transcription and, above a certain threshold the SCR/ HPI complex will dominate, thus furning SCR into a repressor. CEl, cortex/endoexpression pattern of MGP, a common target of SCR and LHP1.

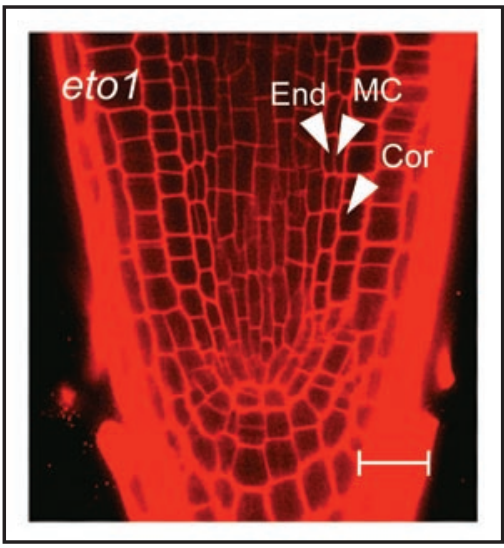

Figure 2. Confocal microscope image of the root of a 6 day-old eto 1 seedling, showing middle cortex (MC) formation. End, endodermis; Cor, cortex. Scale bar: $20 \mu \mathrm{m}$.

a histone deacetylase inhibitor. Moreover, although GA, ABA and SPY antagonize each other in many aspects of development, it is likely that they act in a similar manner in cortex proliferation with epigenetic regulation being the unifying principle. Interestingly, a recent report showed that AGO1, a major player in the small interfering RNA (siRNA) biogenesis pathway, ${ }^{13}$ also plays a role in cortex proliferation. ${ }^{14}$ Thus, it seems likely that epigenetic regulation is the common basis for the various pathways in cortex proliferation. To test this hypothesis, we need to identify the genes that control cortex cell division and examine their histone modification status. 
There is evidence that at least some of the components of the mechanisms regulating cortex proliferation in the Arabidopsis root are utilized as well in other plant species. In potato (Solanum tuberosum), for instance, the tuber forms from cortex and pith cells in the underground stem. ${ }^{15}$ Although a number of environmental cues such as photoperiod, nitrogen and temperature affect tuberization, they all appear to exert their effects by modulating endogenous GA levels in the plants. ${ }^{15}$ In Lotus japonica, GA inhibits nodule formation, which is largely a process of cortex proliferation. ${ }^{16}$ However, it is presently unclear whether other pathways are also conserved. Ethylene promotes MC formation in the Arabidopsis root, but it does not seem to affect potato tuberization. ${ }^{15}$ Despite a conserved role of SHR and SCR in defining a single layer of endodermis in land plants, ${ }^{8}$ most plants have multiple layers of cortex, suggesting that SCR homologs in these species may not repress cell division. However, it is noteworthy that the SHR and SCR genes have duplicated in these plants, which could dramatically alter the SHR/SCR regulatory pathway. Further studies are warranted to clarify whether this reflects differences between different organs or species.

\section{References}

1. Cui H, Benfey PN. Interplay between SCARECROW, GA and LIKE HETEROCHROMATIN PROTEIN 1 in ground tissue patterning in the Arabidopsis root. Plant J 2009; In press; DOI: 10.1111/j.1365-313X.2009.03839.x.

2. Dolan L, Janmaat K, Willemsen V, Linstead P, Poethig S, Roberts K, Scheres B. Cellular organisation of the Arabidopsis thaliana root. Development 1993; 119:71-84.

3. Paquette AJ, Benfey PN. Maturation of the ground tissue of the root is regulated by gibberellin and SCARECROW and requires SHORT-ROOT. Plant physiology 2005; 138:636-40.

4. Di Laurenzio L, Wysocka-Diller J, Malamy JE, Pysh L, Helariutta Y, Freshour G, et al. The SCARECROW gene regulates an asymmetric cell division that is essential for generating the radial organization of the Arabidopsis root. Cell 1996; 86:423-33.

5. Helariutta Y, Fukaki H, Wysocka-Diller J, Nakajima K, Jung J, Sena G, et al. The SHORT-ROOT gene controls radial patterning of the Arabidopsis root through radial signaling. Cell 2000; 101:555-67.

6. Turck F, Roudier F, Farrona S, Martin-Magniette ML, Guillaume E, Buisine N, et al. Arabidopsis TFL2/LHP1 specifically associates with genes marked by trimethylation of histone H3 lysine 27. PLoS Genet 2007; 3:86.

7. Zhang X, Germann S, Blus BJ, Khorasanizadeh S, Gaudin V, Jacobsen SE. The Arabidopsis LHP1 protein colocalizes with histone H3 Lys27 trimethylation. Nat Struct Mol Biol 2007; 14:869-71.

8. Cui H, Levesque MP, Vernoux T, Jung JW, Paquette AJ, Gallagher KL, et al. An evolutionarily conserved mechanism delimiting SHR movement defines a single layer of endodermis in plants. Science 2007; 316:421-5.

9. McGinnis KM, Thomas SG, Soule JD, Strader LC, Zale JM, Sun TP, Steber CM. The Arabidopsis SLEEPY1 gene encodes a putative F-box subunit of an SCF E3 ubiquitin ligase. Plant Cell 2003; 15:1120-30.

10. Swain SM, Tseng TS, Thornton TM, Gopalraj M, Olszewski NE. SPINDLY is a nuclearlocalized repressor of gibberellin signal transduction expressed throughout the plant. Plant Physiol 2002; 129:605-15.

11. Yoshida H, Nagata M, Saito K, Wang KL, Ecker JR. Arabidopsis ETO1 specifically interacts with and negatively regulates type 21 -aminocyclopropane-1-carboxylate synthases. BMC Plant Biol 2005; 5:14.

12. Yang X, Zhang F, Kudlow JE. Recruitment of O-GlcNAc transferase to promoters by corepressor mSin3A: coupling protein O-GlcNAcylation to transcriptional repression. Cell 2002; 110:69-80.

13. Mallory AC, Elmayan T, Vaucheret H. MicroRNA maturation and action-the expanding roles of ARGONAUTEs. Curr Opin Plant Biol 2008; 11:560-6.

14. Miyashima S, Hashimoto T, Nakajima K. ARGONAUTE1 acts in Arabidopsis root radial pattern formation independently of the SHR/SCR pathway. Plant Cell Physiol 2009; 50:626-34.

15. Jackson SD. Multiple signaling pathways control tuber induction in potato. Plant Physiol 1999; 119:1-8.

16. Maekawa T, Maekawa-Yoshikawa M, Takeda N, Imaizumi-Anraku H, Murooka Y, Hayashi M. Gibberellin controls the nodulation signaling pathway in Lotus japonicus. Plant J 2009; 58:183-94. 